\title{
747.
}

\section{NOTE ON THE DEGENERATE FORMS OF CURVES.}

[From Salmon's Higher Plane Curves, (3rd ed., 1879), pp. 383-385.]

Some remarks may be added as to the analytical theory of the degenerate forms of curves. As regards conics, a line-pair can be represented in point-coordinates by an equation of the form $x y=0$; and reciprocally a point-pair can be represented in linecoordinates by an equation $\xi \eta=0$, but we have to consider how the point-pair can be represented in point-coordinates: an equation $x^{2}=0$ is no adequate representation of the point-pair, but merely represents (as a two-fold or twice repeated line) the line joining the two points of the point-pair, all traces of the points themselves being lost in this representation: and it is to be noticed, that the conic, or two-fold line $x^{2}=0$, or say $(\alpha x+\beta y+\gamma z)^{2}=0$ is a conic which, analytically, and (in an improper sense) geometrically, satisfies the condition of touching any line whatever; whereas the only proper tangents of a point-pair are the lines which pass through one or other of the two points of the point-pair.

The solution arises out of the notion of a point-pair, considered as the limit of a conic, or say as an indefinitely flat conic; we have to consider conics certain of the coefficients whereof are infinitesimals, and which, when the infinitesimal coefficients actually vanish, reduce themselves to two-fold lines; and it is, moreover, necessary to consider the evanescent coefficients as infinitesimals of different orders. Thus consider the conics which pass through two given points, and touch two given lines (four conditions); take $y=0, z=0$ for the given lines, $x=0$ for the line joining the given points, and $(x=0, y-\alpha z=0),(x=0, y-\beta z=0)$ for the given points; the equation of a conic satisfying the required conditions and containing one arbitrary parameter $\theta$, is

$$
x^{2}+2 \theta x y+2 \theta \sqrt{ }(\alpha \beta) x z+\theta^{2}(y-\alpha z)(y-\beta z)=0 ;
$$


or, what is the same thing,

$$
\{x+\theta y+\theta \sqrt{ }(\alpha \beta) z\}^{2}-\theta^{2}(\alpha+\beta) y z=0 ;
$$

and this equation, considering therein $\theta$ as an infinitesimal, say of the first order, represents the flat conic or point-pair composed of the two given points. Comparing with the general equation

$$
\left(a, b, c, f, g, h \gamma_{\chi} x, y, z\right)^{2}=0,
$$

we have

$$
a=1, \quad b=\theta^{2}, \quad c=\theta^{2} \alpha \beta, \quad f=-\frac{1}{2} \theta^{2}(\alpha+\beta), \quad g=\theta \sqrt{ }(\alpha \beta), \quad h=\theta,
$$

viz. $a$ being taken to be finite, we have $g$ and $h$ infinitesimals of the first order; $b, c, f$ infinitesimals of the second order; and the four ratios $\sqrt{ }(b): \sqrt{ }(c): \sqrt{ }(f): g: h$ are so determined as to satisfy the prescribed conditions.

Observe that the flat conic, considered as a conic passing through the two given points and touching the two given lines, is represented by a determinate equation, viz. considering the condition imposed upon $\theta(\theta=$ infinitesimal $)$ as a determination of $\theta$, the equation is a completely determinate one; but considering the flat conic merely as a conic passing through the two given points, the equation would contain two arbitrary parameters, determinable if the flat conic was subjected to the condition of touching two given lines, or to any other two conditions.

Generally, we may consider the equation of a curve of the order $n$; such equation containing certain infinitesimal coefficients and, when these vanish, reducing itself to a composite equation $P^{a} Q^{\beta} \ldots=0$; the equation in its original form represents a curve which may be called the penultimate curve. Consider the tangents from an arbitrary point to the penultimate curve; when this breaks up, the system of tangents reduces itself to (1) the tangents from the fixed point to the severai component curves $P=0, Q=0$, \&c. respectively; (2) the lines through the singular points of these same curves respectively; (3) the lines through the points of intersection $P=0, Q=0$, \&c. of each two of the component curves; these points, each reckoned a proper number of times, are called "fixed summits"; (4) the lines from the fixed point to certain determinate points called "free summits" on the several component curves $P=0$, $Q=0$, \&c. respectively. We have thus a degenerate form of the $n$-thic curve, which may be regarded as consisting of the component curves, each its proper number of times, and of the foregoing points called summits, and is consequently only inadequately represented by the ultimate equation $P^{a} Q^{\beta} \ldots=0$; the number and distribution of the summits is not arbitrary, but is regulated by laws arising from the consideration of the penultimate curve, and there are of course for any given value of $n$ various forms of degenerate curve, according to the different ultimate forms $P^{a} Q^{\beta} \ldots=0$, and to the number and distribution of the summits on the different component curves. The case of a quartic curve having the ultimate form $x^{2} y^{2}=0$ has been considered by Cayley, Comptes Rendus, t. Lxxiv. p. 708 (March, 1872), [515], who states his conclusion as follows: 
"there exists a quartic curve the penultimate of $x^{2} y^{2}=0$, with nine free summits, three of them on one of the lines (say the line $y=0$ ), and which are three of the intersections of the quartic by this line (the fourth intersection being indefinitely near to the point $x=0, y=0$ ), six situate at pleasure on the other line $x=0$; and three fixed summits at the intersection of the two lines." Other forms have been considered by Dr Zeuthen, Comptes Rendus, t. Lxxv. pp. 703 and 950 (September and October, 1872), and some other forms by Zeuthen; the whole question of the degenerate forms of curves is one well deserving further investigation.

The question of the number of cubic curves satisfying given elementary conditions (depending as it does on the consideration of the degenerate forms of these curves) has been solved by Maillard and Zeuthen; that of the number of quartic curves has been solved by Dr Zeuthen. 